\title{
Automatic control of the NMB level in general anaesthesia with a switching total system mass control strategy
}

\author{
Miguel Teixeira . Teresa Mendonça . \\ Paula Rocha • Rui Rabiço
}

Received: date / Accepted: date

\begin{abstract}
This paper presents a model based switching control strategy to drive the neuromuscular blockade (NMB) level of patients undergoing general anesthesia to a predefined reference. A single-input single-output Wiener system with only two parameters is used to model the effect of two different muscle relaxants, atracurium and rocuronium, and a switching controller is designed based on a bank of total system mass control laws. Each of such laws is tuned for an individual model from a bank chosen to represent the behavior of the whole population. The control law to be applied at each instant corresponds to the model whose NMB response is closer to the patient's response. Moreover a scheme to improve the reference tracking quality based on the analysis of the patient's response, as well as, a comparison between the switching strategy and the Extended Kalman Filter EKF technique are presented. The results are illustrated by means of several simulations, where switching shows to provide good results, both in theory and in practice, with a desirable reference tracking. The reference tracking improvement technique is able to produce a better reference tracking. Also, this technique showed a better performance than the (EKF). Based on these results, the switch-
\end{abstract}

M. Teixeira

Faculdade de Ciências da Universidade do Porto

Rua Campo Alegre 687, 4169-007, Porto

E-mail: teixeira.mrt@fc.up.pt

T. Mendonça

Faculdade de Ciências da Universidade do Porto

Rua Campo Alegre 687, 4169-007, Porto

E-mail: tmendo@fc.up.pt

P. Rocha

Faculdade de Engenharia da Universidade do Porto

Rua Dr. Roberto Frias, 4200-465 Porto, Portugal

E-mail: mprocha@fe.up.pt

R. Rabiço

Department of Anesthesiology

Unidade Local de Saúde de Matosinhos - Hospital Pedro Hispano

Rua Dr. Eduardo Torres, 4464-513 Senhora da Hora

E-mail: rrabico@gmail.com 
ing control strategy with a bank of total system mass control laws proved to be robust enough to be used as an automatic control system for the NMB level.

Keywords Automatic Delivery System · Switching Control · Positive Control System · Minimally Parameterized Parsimonious Models

\section{Introduction}

Anesthesia can be defined as a drug-induced reversible state where three variables must be controlled: hypnosis, analgesia, and areflexia. Areflexia is defined as the lack of movement. It is induced and maintained by the administration of muscle relaxants, such atracurium and rocuronium, whose goal is to achieve an appropriate level of paralysis during surgical procedures. The neuromuscular blockade (NMB) can be clinically measured by electrical stimulation of the adductor pollicis muscle in the patient's hand where the NMB level corresponds to the first single response calibrated by a reference twitch.

The NMB level can be modeled by pharmacokinetics/pharmacodynamics (PK/ PD) models that relate in a first step the administered drug dose with the drug concentration in the relevant part of the patient's body, known as the effect compartment, and then relate, in a second step, the effect concentration with the actual drug effect (here, the NMB level).

Compartmental systems are widely used to model the PK/PD of intravenously administered drugs [4]. A compartmental system is a system that has a finite number of homogeneous, well-mixed subsystems, called compartments that exchange material among them and with the environment. These interchanges are described by laws that take into account the transfer of material, accumulation and elimination in each compartment and to the environment.

When compared with manual drug administration, automatic control strategies may carry considerable advantages like avoiding under or overdosing. In order to implement automatic control, reliable models are needed for the patients. This implies setting up a suitable class of parameterized models as well as automatic parameterized identification strategies based on those models able to identify the patient parameters taking into account the inter- and intraindividual variability. If such controls could be successfully achieved, the drawback of using standardized procedures in drug administration based on population studies can be overcome.

This paper presents a control strategy for the NMB level using a total system mass control law for a new minimally parameterized parsimonious (MPP) compartmental model [7] describing the effect of the muscle relaxant rocuronium in the NMB level [7]. The total system mass control law for feedback stabilization of compartmental systems of [3] is used and a switching control strategy is applied in order to perform the parameter identification needed to obtain the control input. A scheme to improve the reference tracking quality based on the analysis of the patient's steady-state response is presented. Also, a comparison between the technique here presented with the $E K F$ technique is shown.

The contents of the paper are as follows: Section 2 presents the model for neuromuscular blockade control, describing the MPP Wiener model for the NMB level and the total system mass control law used to drive the NMB level to the desired reference. In Section 3 the switching control strategy used to define the 
control law is described together with the scheme to improve the reference tracking, while in Section 4 the simulation results of the implementation of such control strategy are presented. In Section 5 a comparison between the switching strategy and the EKF technique is performed. In Section 6 the pratical results of the implementation of such control strategy are presented. Finally, in Section 7 the conclusions are drawn.

\section{A model for neuromuscular blockade control}

\subsection{Minimally Parameterized Parsimonious NMB model}

In this paper we consider the model proposed in [7] for the effect of a muscle relaxant in the neuromuscular blockade level. This is a Wiener model whose linear part relates the administered drug dose, $u(t)$, to the drug effect concentration, $C_{e}(t)$, and is given by a transfer function of the form:

$$
h(s)=\frac{\left(k_{1} k_{2} k_{3} \alpha^{3}\right)}{\left(s+k_{1} \alpha\right)\left(s+k_{2} \alpha\right)\left(s+k_{3} \alpha\right)},
$$

where $k_{1}, k_{2}, k_{3}$ are fixed, and $\alpha$ is a patient dependent parameter. According to [7], where a brute force search on the real database was performed, the values of $k_{1}, k_{2}, k_{3}$ are fixed to $1,4,10$, respectively.

The nonlinear part of the model relates the drug effect concentration, $C_{e}(t)$, to the produced neuromuscular blockade, $r(t)$, and is given by the Hill equation $[[9]]$ :

$$
r(t)=\frac{100 C_{50}^{\gamma}}{C_{50}^{\gamma}+C_{e}(t)^{\gamma}}
$$

where $\gamma$ is a patient dependent parameter. Moreover $C_{50}$ does not represent as usual the concentration at half of the maximal drug-related effect, but is rather a modified value that incorporates the gain of the linear part. This parameter is taken to be fixed and equal to 3.24 for atracurium and 1 for rocuronium. Here $r(t)$ varies in a scale from $0 \%$ to $100 \%$ where 0 corresponds to full paralysis and 100 to full muscular activity.

In order to apply the proposed control strategy the transfer function $h(s)$ is realized by means of a 3-compartmental state-space model of the form:

$$
\left\{\begin{array}{l}
\dot{x}(t)=A(\alpha)(t)+B(\alpha) u(t) \\
C_{e}(t)=C x(t)
\end{array}\right.
$$

where $A(\alpha)=\alpha A, B(\alpha)=\alpha B$, with

$$
A=\left[\begin{array}{ccc}
-k_{3} & 0 & 0 \\
k_{2} & -k_{2} & 0 \\
0 & k_{1} & -k_{1}
\end{array}\right], \quad B=\left[\begin{array}{c}
k_{3} \\
0 \\
0
\end{array}\right] \quad \text { e } \quad C=\left[\begin{array}{lll}
1 & 0 & 0
\end{array}\right]
$$


2.2 Total system mass control law

It has been shown in [1] that the following positive control law proposed in [3] for total mass control:

$$
\left\{\begin{array}{l}
u(t)=\max (0, \tilde{u}(t)) \\
\tilde{u}(t)=\left(\sum b_{i}(\alpha)\right)^{-1}\left[(111) A(\alpha) x(t)+\lambda\left(M(x)-M^{*}\right)\right]
\end{array}\right.
$$

not only leads the total system mass $M(x(t))=\sum_{i=1}^{3} x_{i}(t)$ to a reference value $M^{*}$, but also leads the effect concentration, $C_{e}(t)$, to the reference value $C_{e}^{*}=$ $M^{*} / 3$. This can be used to control the NMB level in the following way. Given a desired reference value $r^{*}$ for the NMB, compute the corresponding reference level for the effect concentration $C_{e}^{*}$ by inverting the Hill equation, i.e.

$$
C_{e}^{*}=\left(\frac{100}{r^{*}}-1\right)^{1 / \gamma} \times C_{50}
$$

and set $M^{*}=3 C_{e}^{*}$ in the control law (4). This guarantees that the NMB follows the desired reference level, $r^{*}$.

Note however that this control strategy strongly rests on knowledge of the patient dependent parameter $\gamma$, which is unknown in practical cases. In order to overcome this situation, a switching control strategy is introduced in the next section.

\section{Switching control strategy}

\subsection{Switching control}

In order to take into account parameter variability, the following model based switching control strategy is adopted. A bank $\mathcal{P}=\left\{\mathcal{P}_{1}, \ldots, \mathcal{P}_{N}\right\}$ of representative Wiener models is considered, together with a bank of corresponding controllers $\mathcal{K}=\left\{\mathcal{K}_{1}, \ldots, \mathcal{K}_{N}\right\}$ each of them tuned according to what has been explained in the previous section. More concretely if $\mathcal{P}_{i}=\mathcal{P}\left(\alpha_{i}, \gamma_{i}\right)$ then $\mathcal{K}_{i}$ produces the control law:

$$
u_{i}(t)=\max \left(0, \tilde{u}_{i}(t)\right)
$$

with

$$
\tilde{u}_{i}(t)=\frac{1}{k_{3}}\left[\left(k_{3}-k_{2}\right) x_{1}+\left(k_{2}-k_{1}\right) x_{2}+k_{1} x_{3}\right]-\frac{\lambda}{k_{3} \alpha_{i}}\left(M(t)-M_{i}^{*}\right) .
$$

For the action of atracurium the bank $\mathcal{P}$ was built taking into account real data acquired during surgeries. Based on that data the joint distribution for the parameters $(\alpha, \gamma)$ is considered as follows [6]:

$$
(\ln (\alpha), \ln (\gamma)) \sim B N(\mu, \Sigma),
$$

$$
\text { where } \mu=\left[\begin{array}{c}
-3.2870 \\
0.9812
\end{array}\right] \text { is the mean vector and } \Sigma=\left[\begin{array}{cc}
0.0250 & -0.0179 \\
-0.0179 & 0.1196
\end{array}\right]
$$



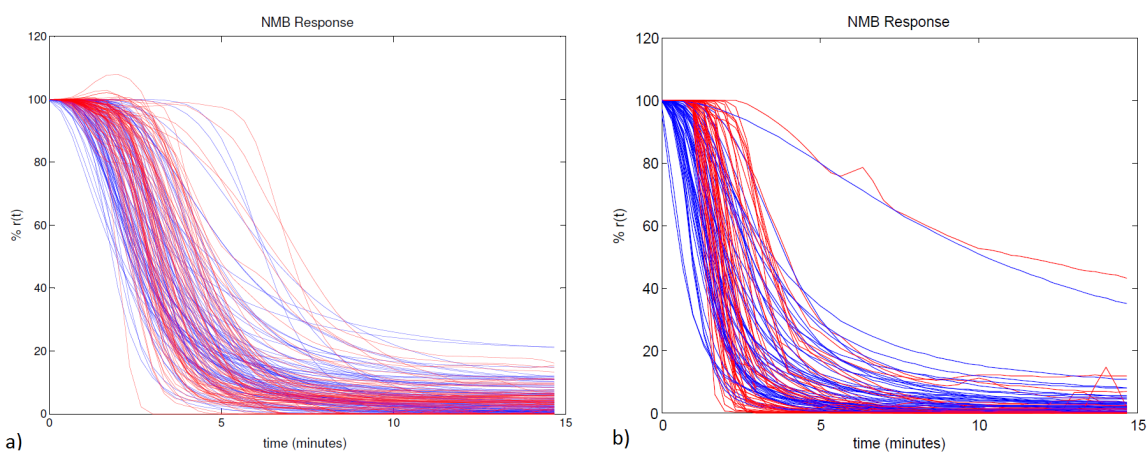

Fig. 1 a) - Red: real NMB responses acquired during surgery performed with atracurium; these responses may be corrupt by measurment noise and sensor faults. Blue: NMB responses of the atracurium models from the bank $P$. In both NMB responses an initial bolus of $500 \mu \mathrm{g} / \mathrm{kg}$ was administered. b) - Red: real NMB responses acquired during surgery performed with rocuronium; these responses may be corrupt by measurment noise and sensor faults. Blue: NMB responses of the rocuronium models from the bank $P$. The doses applied to the real cases were applied to the respective models of the bank.

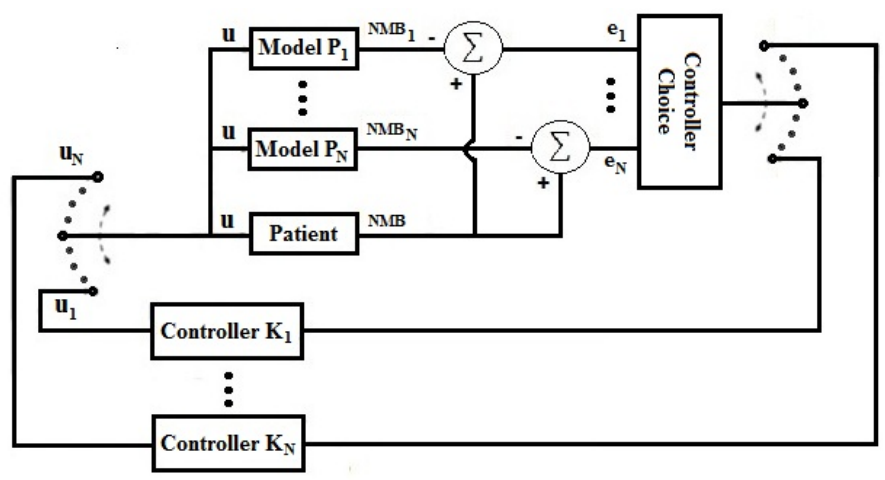

Fig. 2 Switching Control Strategy

is the covariance matrix. The standard bank of representative Wiener models was generated from this distribution (See Fig. 1) as can be seen in Fig 1, the responses of the models in the bank replicate quite satisfactorily the patient responses during surgery.

A similar study of the action of rocuronium is still being performed, and at this point only a set of real data acquired during 50 surgeries is available. For each of these real cases, the parameters $\alpha$ and $\gamma$ were identified with the Prediction Error Method, and the corresponding models were taken as the standard bank of models to perform switching control.

The switching controller computes at each instant the "nearest model" to the patient and applies the corresponding control input to the patient. Proximity is here measured by the cumulative quadratic error between the patient response and the responses of each of the models in the bank (see Fig. 2). 
3.2 Reference tracking improvement

Since the parameters $(\alpha, \gamma)$ as well as the real value for $M^{*}$ for the real patient are unknown it is expected to have a reference steady state tracking error. In order to overcome this drawback a scheme to improve the tracking quality is here proposed. This strategy relying on the NMB response at steady state performs the online tunning of the patient parameter $\gamma$.

For this purpose the input applied to the patient must be at steady state, $u_{S S}$, and the corresponding steady state NMB level response, $r_{S S}$, is supposed to be reached. At this stage the following equation described in [2] and suitably adapted to this model structure is applied for obtaining the correct value, $\gamma^{*}$, of $\gamma$ :

$$
\gamma^{*}=\frac{\ln \left(\frac{100}{r_{S S}}-1\right)}{\ln \left(\frac{u_{S S}}{C_{50}}\right)} .
$$

It turns out that a correct identification of this parameter will lead to the improvement of the control law, since the value of $M^{*}=3 C_{e}^{*}$ only depends on the parameter $\gamma(5)$. After this step the parameter $\gamma$ is fixed as $\gamma^{*}$ and each control law $\tilde{u}_{i}$ is adapted as:

$$
\begin{aligned}
& u(t)=\max (0, \tilde{u}(t)) \\
& \tilde{u}_{i}(t)=\frac{1}{k_{3}}\left[\left(k_{3}-k_{2}\right) x_{1}+\left(k_{2}-k_{1}\right) x_{2}+k_{1} x_{3}\right]-\frac{\lambda}{k_{3} \alpha_{i}}\left(M(t)-M^{*}\right) .
\end{aligned}
$$

Moreover the choice of the control law to be applied at each instant is now made based on the cumulative quadratic error between the $C_{e}$ response of the patient and the response $C_{e}^{i}$ of the $i$-th model in the bank $(i=1, \ldots, N)$. This cumulative error is obtained since the instant where the control of the patient NMB level starts using (11).

\section{Simulation Results}

In order to assess the performance of the proposed switching control strategy a bank of one hundred nonlinear dynamic models for atracurium and a bank of fifty nonlinear dynamic models for rocuronium were used to describe a wide range of patient dynamics. According to the muscle relaxant used in the sequel the bank $\mathcal{P}$ will consist of the models of the action of the corresponding drug.

In each simulation, a model assumed to describe the patient is taken out from the model bank and the corresponding controller is also removed from the controller bank. The control law applied at each time instant is the one produced by the controller corresponding to the model with the most similar response to the patient response.

This can be summarized in the following steps:

Preparation: A model $\mathcal{P}_{j}$ from the bank $\mathcal{P}$ is chosen as describing the real patient dynamics.

Step $1 t=0$ : A standard bolus of $500 \mu \mathrm{g} / \mathrm{kg}$ for atracurium or $600 \mu \mathrm{g} / \mathrm{kg}$ for rocuronium is given to the patient.

Step $20<t<t^{*}$ (before recovery from the initial bolus): The input remains zero. 

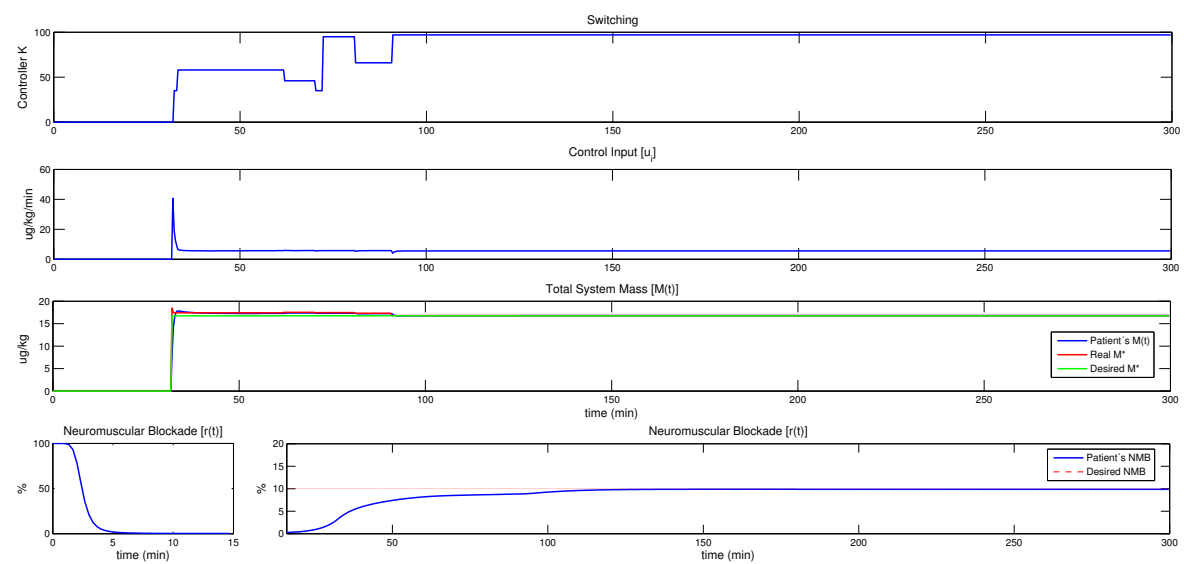

Fig. 3 Results obtained for atracurium $\mathcal{P}=M_{59}$ with $\alpha=0,0474$ and $\gamma=4,0478$ presenting a good reference tracking.

Step $3 t=t^{*}$ (the time instant $t^{*}$ is detected automatically by the OLARD algorithm introduced in [8] and corresponds to the recovery from the initial bolus): A random controller from bank $\mathcal{K}$ is chosen in order to start the control of the NMB response of the patient.

Step $4 t \geq t^{*}$ : A controller $\mathcal{K}_{i}$ is chosen at each time instant based on the minimization of the cumulative error between the patient response and the responses of each of the models in the bank $\mathcal{P} \backslash\left\{\mathcal{P}_{j}\right\}$.

Step 5 (if applied): Corresponds to the reference tracking improvement where $\gamma$ is fixed as $\gamma^{*}$ given by (8), and the selection of the controller $\mathcal{K}_{i}$ is then based on the minimization of the cumulative error between the $C_{e}$ response of the patient and the $C_{e}$ responses of each of the models in the bank $\mathcal{P} \backslash\left\{\mathcal{P}_{j}\right\}$ in order to obtain $\alpha_{i}$ for the control law (11).

The corresponding simulations are described in Figs. 3 to 8. Figs. 3 to 5 represent the simulations for atracurium where Fig. 3 refers to a good case of switching control, and Figs. 4 and 5 represent a poor case of switching control without and with reference tracking improvement, respectively. The other three figures (Figs. 6 to 8 ) describe the switching control strategy applied to the case of rocuronium, where Fig. 6 concerns a good case of switching control and the last two figures, 7 and 8 , refer to a poor case of switching control without and with reference tracking improvement, respectively.

In each of the figures, the upper subplot displays the indices $i$ of the controllers $\mathcal{K}_{i}$ chosen during the control procedure. The subplot below refers to the control input, $u_{i}[\mu \mathrm{g} / \mathrm{kg} / \mathrm{min}]$ which remains zero until the recovery from the initial bolus occurs. The third subplot corresponds to the total system mass for the patient under consideration, $M(t)[\mu \mathrm{g} / \mathrm{kg}]$, (blue line) and to the evolution of the values of $M^{*}$ during control (red line), as well as the real value of $M$ necessary to drive the patient's NMB level to the desired reference of $10 \%$ (green line). Finally the lower subplots show the patient's NMB (blue line) and the NMB control target (red line), 10\% (ref), where the left subplot represents the NMB response to the 

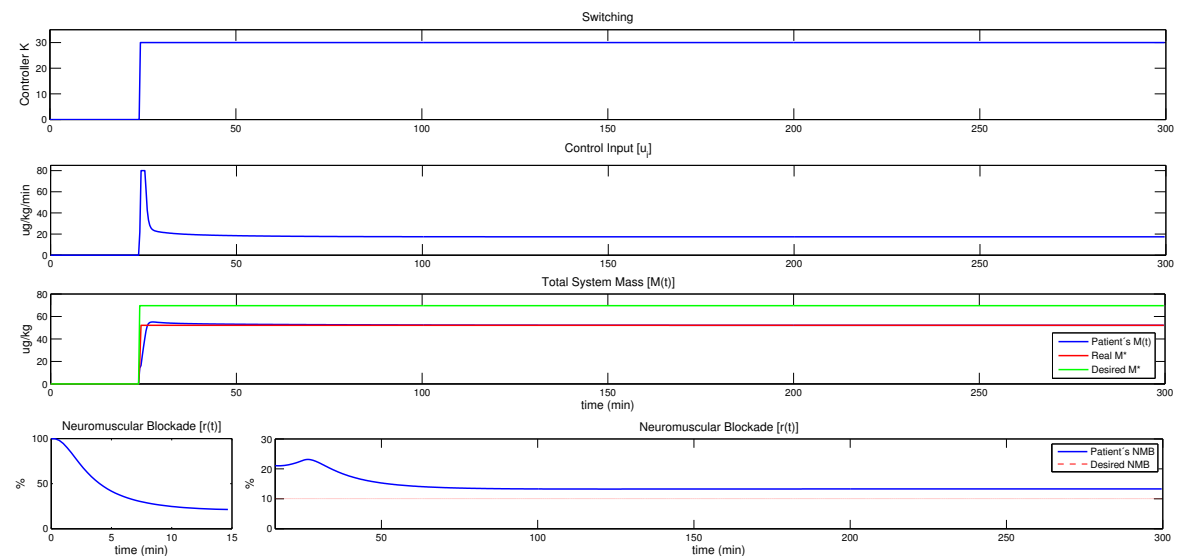

Fig. 4 Results obtained for atracurium $\mathcal{P}=M_{38}$ with $\alpha=0,0345$ and $\gamma=1,1170$ presenting a poor reference tracking.
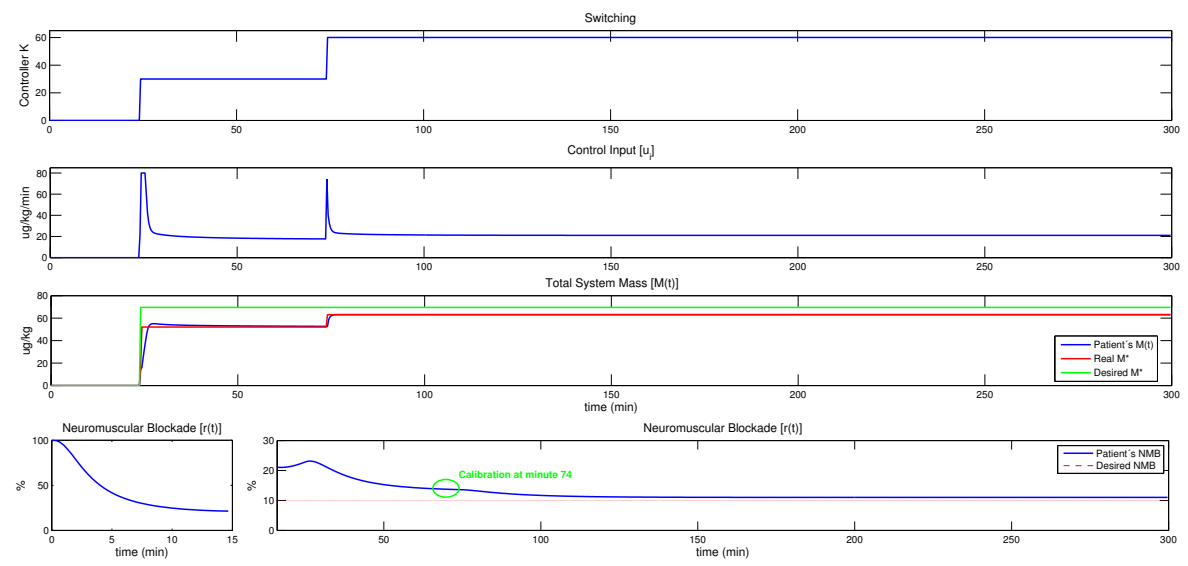

Fig. 5 Results obtained for atracurium $\mathcal{P}=M_{38}$ with $\alpha=0,0345$ and $\gamma=1,1170$ with reference tracking improvement performed at minute 74 .

initial bolus, and the right subplot shows the patient NMB response during the recovery to the initial bolus and the control. All variables are functions of time, expressed in minutes.

Fig. 3 shows a case where the switching control strategy has a good performance. The last active controller is the one corresponding to model 97, and the total system mass tends to $M_{97}^{*}$, which corresponds to a steady state value for the patient's NMB level with an absolute error of 0.14 with respect to the desired reference value of $10 \%$.

Fig. 4 illustrates a case with relatively poor performance, where the last active controller is the one corresponding to model 30 with an absolute steady state error of 3.30 . 

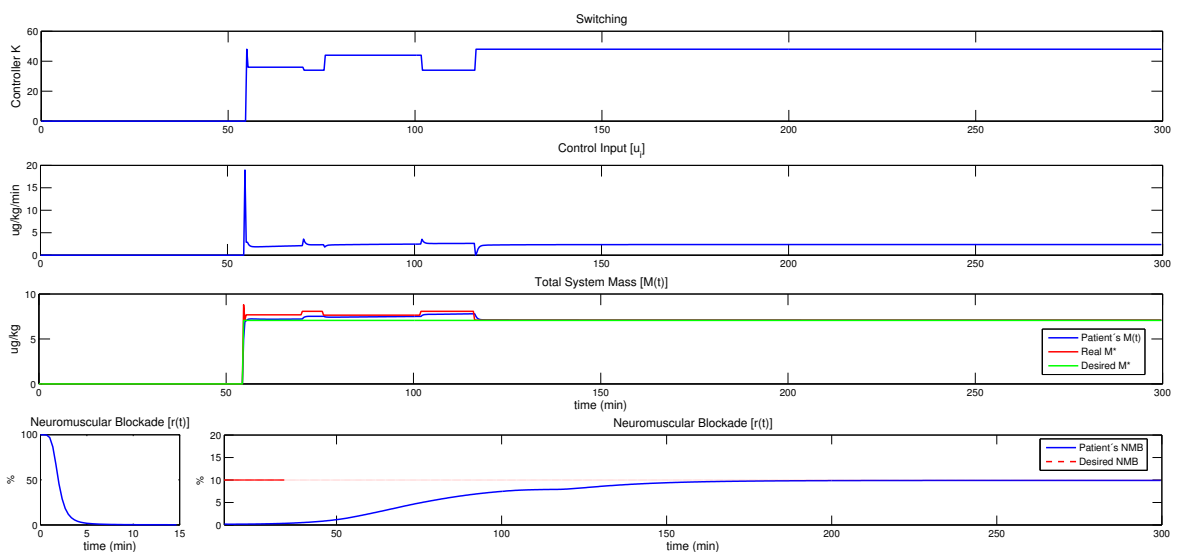

Fig. 6 Results obtained for rocuronium $\mathcal{P}=M_{6}$ with $\alpha=0,0329$ and $\gamma=2,5669$ presenting a good reference tracking.
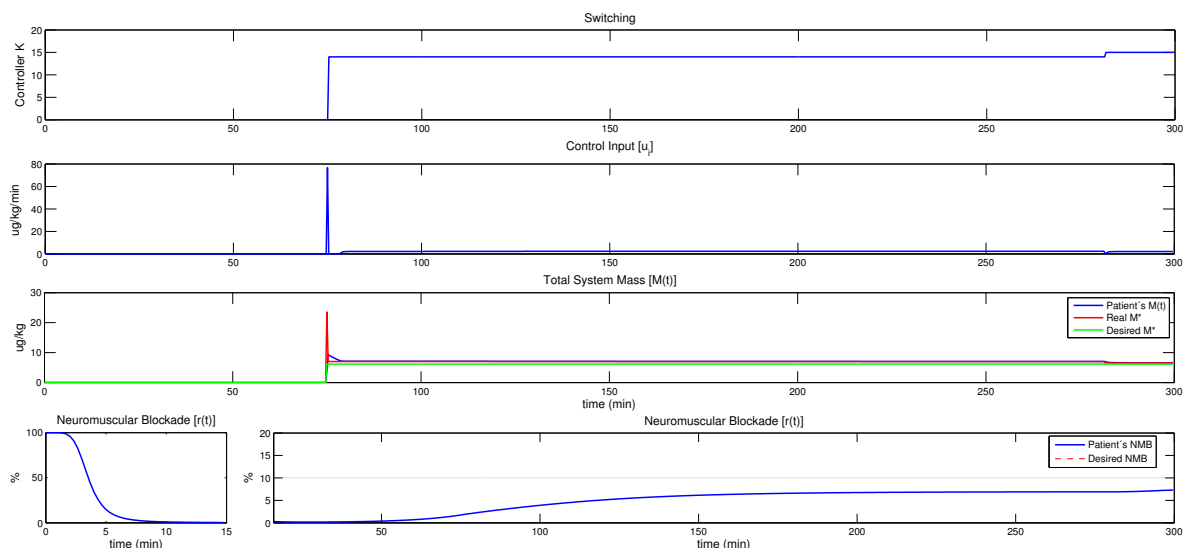

Fig. 7 Results obtained for rocuronium $\mathcal{P}=M_{24}$ with $\alpha=0,0219$ and $\gamma=3,0520$ presenting a poor reference tracking.

Fig. 5 shows the result of the aplication of the reference tracking improvement technique to this case. The calibration was performed at minute 74 , and the absolute steady state error was reduced to only 1.02 .

As for rocuronium, Fig. 6 exhibits a case where the performance of the switching control strategy is good. The last active controller is the one corresponding to model 48 , and the total system mass tends to $M_{48}^{*}$, which corresponds to a steady state value for the patient's NMB level with an absolute error of 0.10 with respect to the desired reference value of $10 \%$.

The case illustrated in Fig. 7 has a worse performance, with a 2.69 absolute steady state error. Again, the application of the reference tracking improvement, 

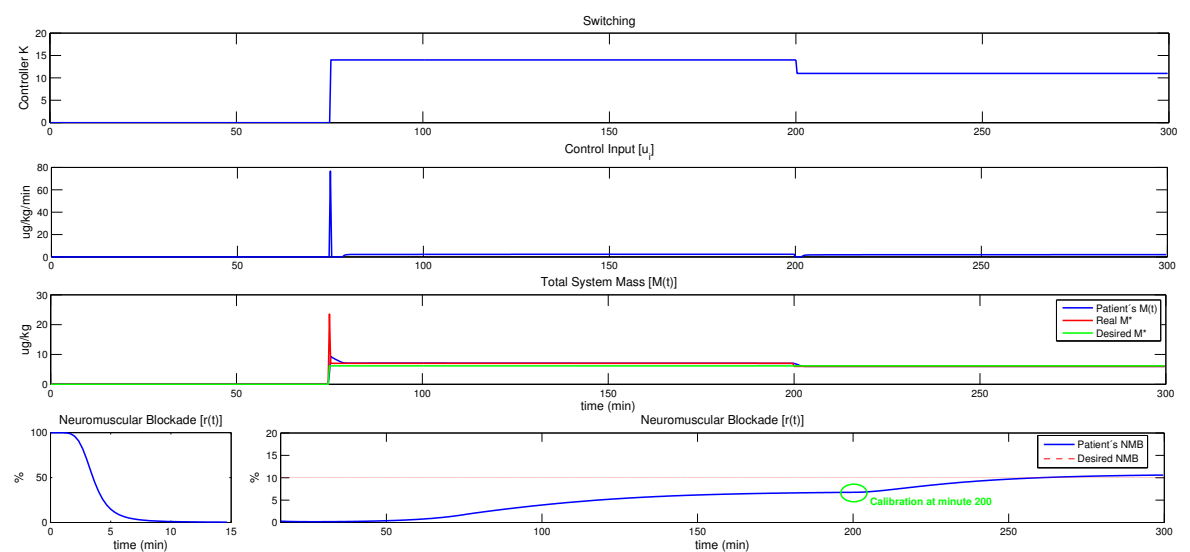

Fig. 8 Results obtained for rocuronium $\mathcal{P}=M_{24}$ with $\alpha=0,0219$ and $\gamma=3,0520$ with reference tracking improvement performed at minute 200 .

here applied at minute 200 , reduces that error, now to a value of only 0.59 , as can be seen in Fig. 8.

Finally it is important to highlight that in the most part of the cases the control signal presents always several switching situations between controllers of the associated bank, mainly during transient period. Moreover, it may be observed that in most cases the effect (NMB) approaches the desired reference profile (ref), despite the occurrence of a difference between both signals, in some cases. This latter situation may occur whenever there are not enough models in the bank that are sufficiently close to the model of the real patient. The scheme proposed in order to improve the reference tracking quality proved to deliver very good results.

\section{Analyzing the switching strategy}

In order to analyze the switching strategy a comparison was made with the $E x$ tended Kalman Filter $(E K F)[5]$. The EKF performs the identification of the model parameters used in the total system mass control law (4) in order to control the NMB level.

For the purpose of obtaining data the bank $\mathcal{P}$ with 100 models for atracurium and the bank $\mathcal{P}$ with 50 models for rocuronium were used and a simulation of 300 minutes was performed for each model, according to what was explained in section 4. Thereafter the two strategies were analyzed using the following as comparison features:

- The normalized drug amount, given by:

$$
\sum_{k=t^{*}}^{t_{\text {end }}} u(k)
$$

where $u(k)(\mu g / k g / \min )$ is the dose of administered drug at step $k$, and $t^{*}$ is the recovery time, detected by the OLARD algorithm [8]. 
- The set-point, which corresponds to the value (in \%) where NMB level stabilizes;

- The settling time, which is the time that patient's NMB level takes to reach the set-point value, with an error not greater than $2 \%$;

- The tracking quality, measured by:

$$
\frac{1}{n} \sum_{k=\text { settlingtime }}^{t_{\text {end }}} \sqrt{\left(r(k)-r_{r e f}\right)^{2}},
$$

where $n=t_{\text {end }}$ - settlingtime, $r(k)$ is the NMB level at step $k$ and $r_{r e f}$ is the desired reference value of $10 \%$.

The results can be visualized in Tables 1 (for atracurium), and 2 (for rocuronium).

Table 1 Comparison between total mass control with Switching and with Extended Kalman Filter $(E K F)$ (atracurium)

\begin{tabular}{|c|c|c|c|c|c|c|c|c|c|}
\hline & & \multicolumn{2}{|c|}{ Total Input $(\mu g / \mathrm{kg})$} & \multicolumn{2}{|c|}{ Set-Point (\%) } & \multicolumn{2}{|c|}{ Settling Time $(\min )$} & \multicolumn{2}{|c|}{ Ref Track Error (\%) } \\
\hline & & Switching & EKF & Switching & EKF & Switching & EKF & Switching & EKF \\
\hline & & & 2999 & 9,96 & 8,87 & 167 & 116 & 0,493 & \\
\hline $\begin{array}{l}\text { Median } \\
\text { Std Deviation }\end{array}$ & & $\begin{array}{r}2504 \\
999\end{array}$ & $\begin{array}{l}2667 \\
1074\end{array}$ & 9,85 & $\begin{array}{r}9,10 \\
0,88\end{array}$ & 155 & 123 & 0,277 & 0,756 \\
\hline $\begin{array}{l}\text { Std. Deviation } \\
\text { Range }\end{array}$ & & $\begin{array}{r}999 \\
3261\end{array}$ & $\begin{array}{l}1074 \\
4823\end{array}$ & $\begin{array}{l}0,86 \\
5,55\end{array}$ & $\begin{array}{l}0,88 \\
4,10\end{array}$ & $\begin{array}{r}64 \\
226\end{array}$ & $\begin{array}{r}29 \\
115\end{array}$ & 0,633 & $\begin{array}{l}0,838 \\
3,720\end{array}$ \\
\hline 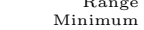 & & $\begin{array}{l}3261 \\
1596\end{array}$ & $\begin{array}{l}4823 \\
1662\end{array}$ & $\begin{array}{l}5,55 \\
6,95\end{array}$ & $\begin{array}{l}4,10 \\
6,05\end{array}$ & $\begin{array}{r}226 \\
69\end{array}$ & $\begin{array}{r}115 \\
51\end{array}$ & $\begin{array}{l}3,240 \\
0,070\end{array}$ & $\begin{array}{l}3,720 \\
0,050\end{array}$ \\
\hline Maximum & & 4857 & 6485 & 12,50 & 10,15 & 296 & 166 & 3,310 & 3,770 \\
\hline Percentiles & $\begin{array}{l}25 \\
75\end{array}$ & $\begin{array}{l}1955 \\
3470\end{array}$ & $\begin{array}{l}2229 \\
3668\end{array}$ & $\begin{array}{r}9,60 \\
10,43\end{array}$ & $\begin{array}{l}8,30 \\
9,53\end{array}$ & $\begin{array}{l}113 \\
218\end{array}$ & $\begin{array}{l}100 \\
135\end{array}$ & $\begin{array}{l}0,162 \\
0,507\end{array}$ & $\begin{array}{l}0,355 \\
1,492\end{array}$ \\
\hline
\end{tabular}

Table 2 Comparison between total mass control with Switching and with Extended Kalman Filter (EKF) (rocuronium)

\begin{tabular}{|c|c|c|c|c|c|c|c|c|c|}
\hline & & \multicolumn{2}{|c|}{ Total Input $(\mu \mathrm{g} / \mathrm{kg})$} & \multicolumn{2}{|c|}{ Set-Point $(\%)$} & \multicolumn{2}{|c|}{ Settling Time $(\min )$} & \multicolumn{2}{|c|}{ Ref Track Error (\%) } \\
\hline & & Switching & EKF & Switching & EKF & Switching & EKF & Switching & EKF \\
\hline Mean & & 6847 & 8293 & 9,90 & 6,33 & 290 & 168 & 0,27 & 3,90 \\
\hline $\begin{array}{l}\text { Median } \\
\text { Stdd Deviation }\end{array}$ & & $\begin{array}{r}6118 \\
2584\end{array}$ & $\begin{array}{r}7629 \\
2872\end{array}$ & 9,90 & 6,38 & 244 & 173 & 0,14 & 3,68 \\
\hline $\begin{array}{l}\text { Std. Deviation } \\
\text { Range }\end{array}$ & & $\begin{array}{r}2584 \\
11315\end{array}$ & $\begin{array}{r}2872 \\
16342\end{array}$ & $\begin{array}{l}0,62 \\
7,30\end{array}$ & $\begin{array}{l}1,64 \\
8,25\end{array}$ & $\begin{array}{l}155 \\
658\end{array}$ & $\begin{array}{r}64 \\
257\end{array}$ & $\begin{array}{l}0,48 \\
3,30\end{array}$ & 1,70 \\
\hline $\begin{array}{l}\text { Range } \\
\text { Minimum }\end{array}$ & & $\begin{array}{r}11315 \\
3750\end{array}$ & $\begin{array}{r}16342 \\
4725\end{array}$ & $\begin{array}{l}7,30 \\
6,10\end{array}$ & $\begin{array}{l}8,25 \\
1,40\end{array}$ & $\begin{array}{r}658 \\
86\end{array}$ & $\begin{array}{r}257 \\
68\end{array}$ & $\begin{array}{l}3,30 \\
0,01\end{array}$ & $\begin{array}{l}8,26 \\
0,49\end{array}$ \\
\hline Maximum & & 15065 & 21067 & 13,40 & $\begin{array}{l}9,65 \\
9,65\end{array}$ & 744 & 325 & 3,31 & 8,75 \\
\hline Percentiles & 25 & 5014 & 6177 & 9,80 & 5,08 & 193 & 111 & 0,06 & 2,66 \\
\hline & 75 & 8101 & 9758 & 10,05 & 7,59 & 340 & 219 & 0,24 & 5,12 \\
\hline
\end{tabular}

In Tables 1 and 2 it can be seen that in average, the total mass control with switching spends less dose than the total mass control with $E K F(E K F+T M C)$. Also the input values in the switching are much closer among themselves than the $E K F+T M C$ input values. This can be seen by the smaller value of the standard deviation, and of the range.

As for the set-point reached with these two techniques, switching presents a better mean NMB value for a desired target of $10 \%$. Moreover the switching has set-point values closer to the average than the $E K F+T M C$, as can be seen by the smaller value of the corresponding standard deviation.

The settling times are higher with the switching than with the $E K F+T M C$. These results can be explained due to the nature of the $E K F+T M C$ strategy, which identifies the parameters to be used in the control law when the recovery to the 
initial bolus is detected; in opposition, as the name suggests, the switching strategy causes changes between controllers over time, and only when the last controller is selected the final set-point is achieved and the settling time is measured.

Finally, analyzing the tracking error (Ref Track Error column) the mean value for switching is much lower than the one verified for the $E K F+T M C$. The same happens to all the other variables except the minimum values with the atracuium models, showing that the switching is better than the $E K F+T M C$. So it can be concluded that the switching strategy has a better reference tracking quality than the $E K F+T M C$ strategy.

In summary, the switching strategy has better results than the $E K F+T M C$ strategy with respect to the total dose used during NMB control, follows better the NMB target with a better reference tracking quality. As for the settling time, the $E K F+T M C$ obtained better results due to the nature of the switching strategy.

\section{Practical Results}

The NMB control strategy presented in this work based on a switching total system mass control law was implemented at the Unidade Local de Saúde de Matosinhos Hospital Pedro Hispano (ULSM-HPH) with the supervision of the anesthesiologist Dr. Rui Rabiço.

The NMB control system applied at the ULSM-HPH consists of:

- A Datex-Ohmeda device (a modular monitoring family with a full line-up of monitors and accessories, a complex solution for anesthesia patient monitoring during surgeries), along with a NMT (neuromuscular transmission) module in order to obtain the NMB level of the patient during surgery;

- A drug delivery system: an Alaris GH syringe pump was used in order to administer the muscle relaxant to the patient;

- A computer: a portable computer with two USB serial ports for connecting the Datex-Ohmeda device and the infusion pump, in order to run the NMB control software.

- Galeno software: a program design to collect all the information produced by the Datex-Ohmeda device, to run the switching control strategy, and to inflict the control input produced by the switching control strategy to the Alaris GH pump infusion.

The protocol used during the surgeries was designed by the anesthesiologist $D r$. Rui Rabiço in accordance with the ethical standards practiced by the ULSM-HPH and the characteristics of the patient:

a) For the hypnotic induction a bolus of propofol was used in order to achieve a quick induction. After this induction the maintenance was made with sevoflurane, a volatile drug.

b) For analgesia a single bolus of fentanil was used at the beginning.

c) Finally, for the arreflexia, the drug rocuronium was used. This drug is nowadays the most widely used due to its special properties, such as: it allows a quick induction of the patient's muscle paralysis and is not affected by renal failure. At the end of the surgery the NMB was reversed either with neostigmine with atropine or with sugammadex, as considered appropriate. 


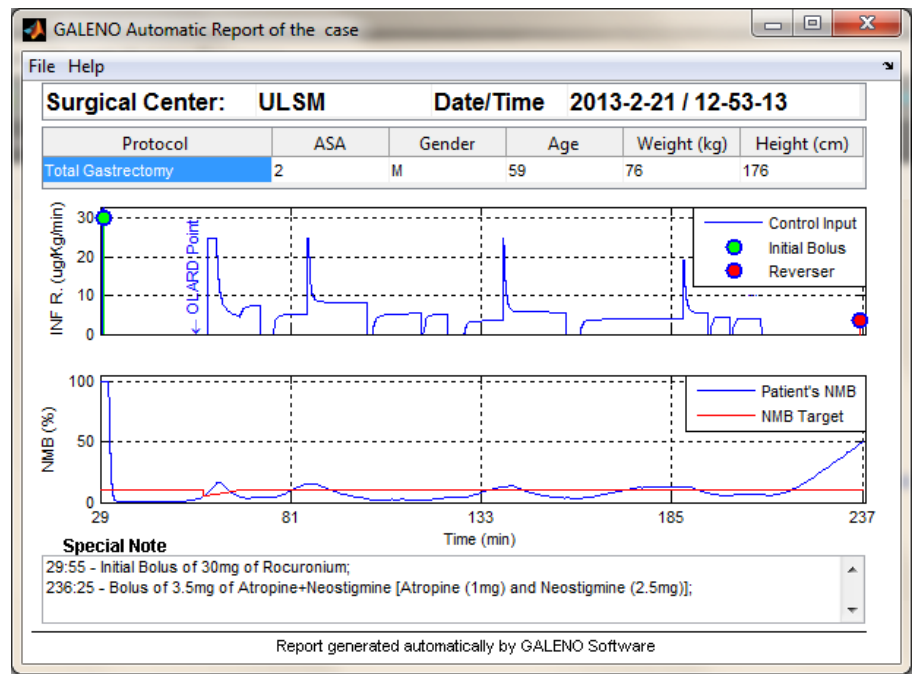

Fig. 9 Real case acquired at ULSM-HPH (2013/02/21 - Case 1).

The process of general anesthesia with NMB control during surgeries can be summarized as follows:

- Step 1 Administration of a fentanil bolus.

- Step 2 Administration of a propofol bolus for induction, followed by sevoflurane administration for maintenance.

- Step 3 Calibration of the NMB Sensor.

- Step $4 t=0$ : Administration of an initial bolus of rocuronium to the patient (Automatic administration performed by the Monitoring and Control in Anesthesia software).

- Step $50<t<t^{*}$ (before recovery from the initial bolus of rocuronium): The control input remains zero.

- Step $6 t=t^{*}\left(t^{*}\right.$ computed by the OLARD algorithm introduced in [8]): A random controller from bank $\mathcal{K}$ is chosen in order to start the control of the NMB response of the patient.

- Step $7 t \geq t^{*}$ : A controller $\mathcal{K}_{i}$ is chosen at each time instant based on the minimization of the cumulative error between the patient response and the responses of each of the models in the bank $\mathcal{P}$.

- Step 8 At the end of the surgery: Administration of NMB reversal drugs, neostigmine with atropine or sugammadex, according to the patient characteristics and his/her NMB level at this stage.

The upper plots of Fig. 9 to 11 contain all the information about the drugs administered to the patient that have effect on the NMB level. The lower plots contain the NMB level of the patient (blue line) and the desired NMB target (red line).

For the real cases collected at ULSM-HPH (Fig 9 to 11), the anesthesiologist considered that the controller performance was satisfactory since the NMB level was kept within a very reasonable interval, and never reached any abnormal values. The controller did not give an overall excessive dose of rocuronium to the patient, 


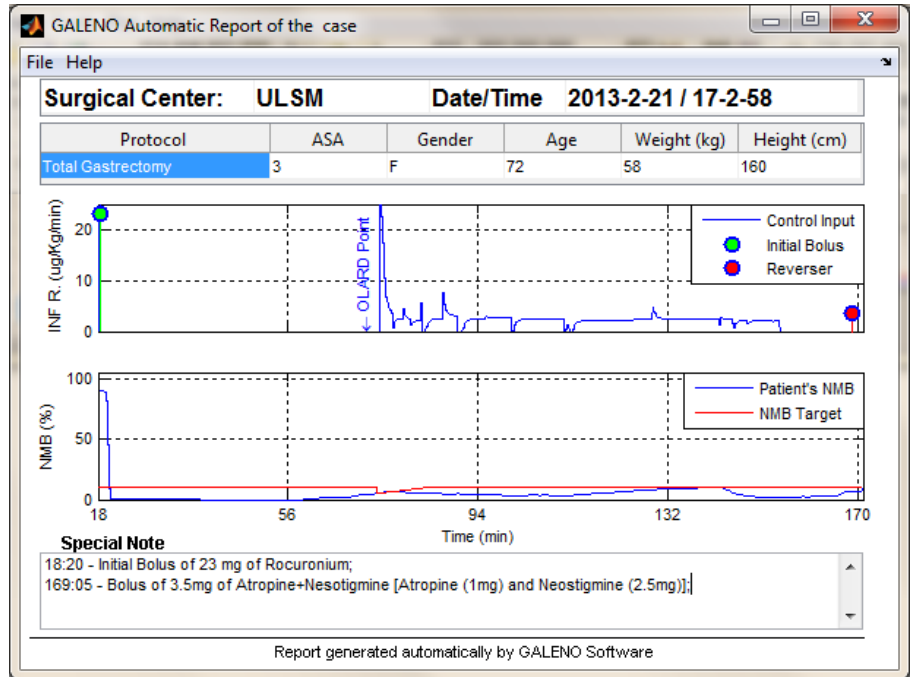

Fig. 10 Real case acquired at ULSM-HPH (2013/02/21 - Case 2).

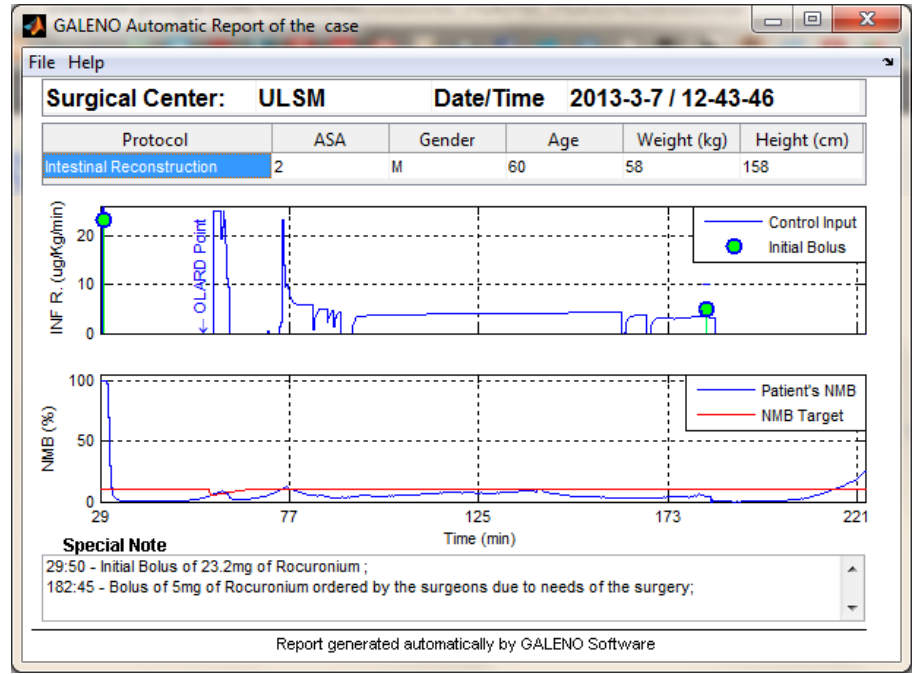

Fig. 11 Real case acquired at ULSM-HPH (2013/03/07 - Case 1).

and no complains were registered either from surgeons or from the anesthesiologist . Therefore one can conclude that, although for practical cases the reference tracking performance was not as high as for simulated cases, the obtained results were still very satisfactory. The application of the reference tracking improvement technique to these cases was not possible once it is very difficult to achieve a steady state input dose with the equivalent steady state response created by such dose. As a final note, the changes that occured in the rocuronium infusion rate during the control are the sign that switching among the controllers in the bank occurred. 
As a conclusion it can be said that the switching strategy shows to provide good results for the NMB level control during general anesthesia. The NMB level was kept between good minimum and maximum values, the patient muscle stiffness was never a point of discussion for the surgeons, and the anesthesiologist never had to perform any manual control on the patient muscle relaxation. Another aspect of this control strategy is its ability to adapt to the intravariablity of a patient dynamics in the course of time.

\section{Conclusion}

This paper presented a new strategy for the control of the NMB level of patients undergoing general anesthesia in the presence of patient-parameter variablity. This consists in using a MPP model previously presented in the literature, which has a compartmental structure. This special structure enables the design of a controller based on the total system mass in order to track a desired NMB level with a switching strategy. In the cases where the reference tracking is not achieved, an improvement of the convergence is perfomed based on the obtained steady state response of the patient. The presented control strategy shows to provide good theoretical results for both atracurium and rocuronium. Also, it is shown that the switching control strategy presents better results than the EKF technique. Finally, this strategy shows to provide good results when applied in real surgeries.

Acknowledgements This work was supported in part by FEDER funds through COMPETE - Operational Programme Factors of Competitiveness ("Programa Operacional Factores de Competitividade") and by Portuguese funds through the Center for Research and Development in Mathematics and Applications and the Portuguese Foundation for Science and Technology ("FCT - Fundação para a Ciência e a Tecnologia"), within project PEst-C/MAT/UI4106/2011 with COMPETE number FCOMP-01-0124-FEDER-022690.

\section{References}

1. Almeida, J., Silva, M. M.; Mendonça, T.; Rocha, P., A Compartmental Model Based Control Strategy for NeuroMuscular Blockade Level, IFAC World Congress, 18, 599-604 (2011)

2. Alonso, H. and Mendonça, T. and Rocha, P., A hybrid method for parameter estimation and its application to biomedical systems, Computer Methods and Programs in Biomedicine, 89, 112-122 (2008)

3. Bastin, G. and Provost, A., Feedback stabilisation with positive control of dissipative compartmental systems, Proceedings of the 15th Internationol Symposium on Mathematical Theory of Networks ans Systems (2002)

4. Godfrey, K., Compartmental models and their application. Academic Press, Salt Lake City (1983)

5. Kalman, R. E., A new approach to linear filtering and prediction problems, Transactions of the ASME Journal of Basic Engineering, 82, 35-45 (1960)

6. Rocha, C. and Mendonça, T. and Silva, M.M., A Simulation Model for Neuromuscular Blockade Induced by Atracurium, Internal Report of CIDMA (2011)

7. Silva, M.M., Wigren, T., and Mendonça, T., Nonlinear identification of a minimal neuromuscular blockade model in anaesthesia, IEEE Trans. Contr. Sys. Tech., 20, 181-188 (2012)

8. Silva, M.M., Sousa, C., Sebastião, R., Gama, J., Mendonça, T., Rocha, P. and Esteves, S., Total Mass TCI driven by Parametric Estimation, 17th Mediterranean Conference on Control and Automation, Makedonia Palace, Thessaloniki, Greece (2009)

9. Weatherley, B., Williams, S., and Neill, E., Pharmacokinetics, pharmacodynamics and doseresponse relationships of atracurium administered i. v., Br. J. Anaesth., 55, 39-45 (1983) 